\title{
EDITORIAL
}

\section{Understanding metastasis}

D espite our impressive knowledge of the cancer genome, we are still relatively in the dark about the origins and even the clinical manifestations of metastasis-essentially, the disease that kills. Tumour heterogeneity and a lack of cell differentiation are at the crux of appreciating the genetic divergence of primary tumours and metastases. In the context of understanding the carcinogenesis continuum, some of our assumptions on mutational divergence during proliferation and how this correlates with tumour growth, clonal expansion, and dissemination, are fraught with inconsistent findings. Two key models of metastasis prevail. The linear progression model posits that the development of metastasis occurs relatively late in a unidirectional continuum at a point when cancer cells have acquired advanced mutational and growth capacities that enable them to colonize distant sites. Conversely, in the parallel progression model, metastases occur relatively early in the development of the primary tumour, and the primary and metastases are considered to evolve independently.

Trying to correlate these two main models of metastasis with clinical data, to support or refute these paradigms, reveals a quagmire. In this issue, Naxerova and Jain tackle some of the more robust as well as the less clear assumptions relating to these models, by correlating phylogenetics and the lineage of cancer with clinical observations. They appropriately begin by stating that we do not know when metastatic precursors occur, nor by what route metastatic cells spread to form widespread disseminated disease. Do metastatic cells develop from the primary tumour and develop independently from each other, or do some metastases give rise to others?

In general, metastasis is assumed to occur shortly before a tumour becomes clinically detectable. In the linear progression model, the evolutionary space between the primary tumour and metastasis is relatively small, and the primary is considered a reasonable surrogate, at a molecular level, for the metastatic tumour. The problem, as highlighted by the authors, is that even in tumours in which the primary and corresponding metastasis are relatively genetically similar, this may not indicate whether the metastases that gave rise to the primary occurred late in the disease process. Furthermore, some tumours that divide and proliferate rapidly and, therefore, accumulate multiple mutations, might also be balanced by high cell death, so in the context of clinical interpretation, the paradigm that is at play becomes difficult to delineate. Conversely, the metastatic clone might not have as high a mutational capacity as assumed, and therefore might retain similarity to the primary, despite an extended period of independent growth. The assumption that the growth rates of primary tumours and metastases are similar, lends support to parallel progression, as linear progression would not explain overt metastases close to the time of primary tumour diagnosis. Imaging analyses generally reveal similar doubling times between primary tumours and metastases; so, if the bulk of the tumour is eradicated by treatment, it would be expected that repopulation to the point of widespread progression would take a long time, yet often this timeframe is remarkably short-a few months. Self-seeding and oligometastases might explain some of the disparities in clinical observations, but do not provide the full picture.

Primary tumour size is also considered to indicate the potential of metastasis risk, although what critical mass of genetic aberrations is needed for metastasis is unclear. The greater the mutational burden of a tumour, the greater its potential to support invasion and survival. Metastasis, however, is complicated by tumour dormancy. When reconstructing the genetic evolution of a tumour by assessing mutations as a surrogate for proliferation, since dormant cells do not divide, the genetic evolutionary clock is suspended. In tumour mass dormancy, the balance between proliferation and cell death is equal, so it is possible that the cells in the tumour that acquire the most mutations might survive; paradoxically, if cells undergoing apoptosis have relatively fewer mutations (because their genetic repertoire is not so corrupt to avoid cell death), this might explain the ability of tumour cells to continue to evolve genetic aberrations, but appear in a seemingly quiescent evolutionary state.

The tumour and normal tissue microenvironment is increasingly recognized as a key element for tumours to thrive. One advantage of a highly proliferating cancer cell (with high mutational burden) is that it might be more detectable by the immune system, so although inducing the dormant state to delay metastases has merit, it might also be a double-edged sword in terms of the interplay with the microenvironment and immune system. Another intrigue is what level of mutational burden and alterations adjacent normal cells undergo during carcinogenesis, and how do the signals they secrete support the expansion of cancer growth. A study published in Nature by Joan Massague and co-authors highlights that therapeutic inhibition of oncogenic drivers in drug-sensitive cells, induces secreted signals that promote the expansion of drug-resistance clones - a finding providing new avenues for further investigation of metastasis.

doi:10.1038/nrclinonc.2015.71

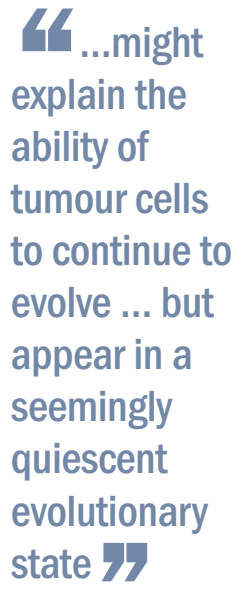

Lisa Hutchinson is the Chief Editor of Nature Reviews Clinical Oncology.

Competing interests The author declares no competing interests. 